Case Report

\title{
A Case of Idiopathic Normal Pressure Hydrocephalus Presenting as Vascular Parkinsonism
}

\author{
Talal Meshal Alumri ${ }^{1}$, Rawan Zeinelabdein Mahgoub ${ }^{2}$, Alawi Aqel Al-Attas ${ }^{3,}$ * \\ ${ }^{1}$ Department of Neurology, Ministry of National Guard - Health Affairs, Riyadh, Saudi Arabia \\ ${ }^{2}$ College of Medicine, Dar Al-Uloom University, Riyadh, Saudi Arabia \\ ${ }^{3}$ Department of Neurology, Prince Sultan Military Medical City, Riyadh, Saudi Arabia
}

\section{Email address:}

Talal.uni@gmail.com (T. M. Alumri), Rawan.mahgoub93@hotmail.com (R. Z. Mahgoub), Alawiattas@yahoo.com (A. A. Al-Attas)

${ }^{*}$ Corresponding author

\section{To cite this article:}

Talal Meshal Alumri, Rawan Zeinelabdein Mahgoub, Alawi Aqel Al-Attas. A Case of Idiopathic Normal Pressure Hydrocephalus Presenting as Vascular Parkinsonism. American Journal of Psychiatry and Neuroscience. Vol. 8, No. 1, 2020, pp. 1-5. doi: 10.11648/j.ajpn.20200801.11

Received: November 24, 2019; Accepted: December 23, 2019; Published: January 6, 2020

\begin{abstract}
Idiopathic normal pressure hydrocephalus (iNPH) is the most common form of hydrocephalus in adults. It is characterized by dilated ventricles and the Hakim triad, which consists of gait disturbance, cognitive impairment, and urinary incontinence. The gold standard treatment for iNPH is shunting, but frequent lumbar punctures (LPs) are helpful for diagnosis and predicting shunting outcome and is considered the treatment of choice when shunting is not available. One condition with similar presentation to iNPH is Vascular parkinsonism (VP), which is characterized by an established relationship between cerebrovascular diseases and the clinical symptoms of Parkinson's disease (PD). VP patients usually present with lower-body parkinsonism, including impaired gait, instability, difficulty with maintaining gait, and frequent episodes of freezing. VP is managed by levodopa followed by transcranial magnetic stimulation then vitamin D. LPs are less effective for VP than iNPH. Our case report shows that iNPH may be misdiagnosed with VP. Here we present a case of a 71-year-old male with a history of previous lacunar strokes who was admitted for possible vascular parkinsonism. Eight months prior to coming to the hospital, he developed gait disturbances; and four months later, he developed urinary symptoms. Upon examination, his gait initiation was hesitant, wide-based, and magnetic with decreased arm swing. The patient scored 20 out of 30 points on the Montreal Cognitive Assessment. The only cranial nerve involvement was a diminished gag reflex. Brain magnetic resonance imaging revealed symmetrically enlarged ventricles with mildly dilated sulci. Routine laboratory testing was normal. Two months of treatment with levodopa did not improve his symptoms but draining $50 \mathrm{~mL}$ of cerebrospinal fluid (CSF) has dramatically improved his gait. A final diagnosis of iNPH was therefore made. To conclude, iNPH and VP are two manageable diseases but the differential diagnosis between them is particularly challenging due to similar clinical and neuroradiological features. We suggest further studies to establish supporting guidelines by using neuroradiological or neurophysiological biomarkers which may help physicians in ameliorate diagnostic accuracy between the two entities.
\end{abstract}

Keywords: Idiopathic Normal Pressure Hydrocephalus, Vascular Parkinsonism, Lumbar Puncture

\section{Introduction}

Idiopathic normal pressure hydrocephalus (iNPH) is the most common form of hydrocephalus in adults. Although clinical demographic data is lacking in the Saudi Arabian population, the average age of onset in other populations is 70 years old, with a male to female ratio of $1: 1$. [1] The clinical manifestation is characterized by the Hakim triad (consisting of gait disturbance, cognitive impairment, and urinary disturbance) along with dilated ventricles. [1] Gait disturbance, which varies in severity, is the earliest symptom, while cognitive and urinary disturbances appear in the later stages. [2-4] Apart from the Hakim triad, examination is unrevealed. Gait disturbances are typically symmetrical, and the presence of lateralization might decrease the likelihood of iNPH. [5] Such gait disturbances and unsteadiness increase 
patients' fall risk. $[6,7]$ The urinary abnormalities of laterstage disease manifest as urgency or increased frequency, usually accompanied by incontinence. [1] In addition to the physical symptoms of iNPH, involvement of prefrontal brain structures can result in cognitive impairment that may render the patient unreliable as a primary historian. [1] Dementia commonly manifests as missing medications or an inability to manage finances. [8]

Diagnosis is based on excluding secondary causes, which include intracerebral haemorrhage, spaceoccupying lesions, and CNS infections. [1] Patients with iNPH usually score above 0.3 (ventriculomegaly) on the Evans ratio, although this method is not specific for iNPH. [1] Other radiological findings include flattened Sylvian fissures and periventricular lesions. [1] Laboratory tests may aid in ruling out secondary causes of hydrocephalus, such as sexually-transmitted diseases. [9, 10] The gold standard for iNPH management is surgical shunting, [11, 12] although the success rate depends on the lumbar puncture (LP) outcome. [13] iNPH patients usually undergo a series of neuropsychological evaluations before undergoing LP, with testing then repeated 3 hours later. A significant improvement in mental status and gait predicts a favourable response to shunt surgery. [14] In centres lacking the resources to perform shunt surgery, the management of choice is frequent LPs. [15]

Vascular parkinsonism (VP) was first described by Critchley in 1929 as atherosclerotic parkinsonism and has since also been called pseudo-atherosclerotic parkinsonism and lower body parkinsonism. [16-18] Characterized by an established relationship between cerebrovascular diseases and the clinical symptoms of Parkinson's disease (PD), VP is estimated to constitute 3$12 \%$ of all cases of parkinsonism. [19-21] Studies have attempted to link vascupathologies to VP. [16-18] For example, Van Swieten et al. showed a possible causative relationship between Binswanger disease and the unilateral cribriform state (enlarged perivascular [Virchow-Robin] spaces) to lower body parkinsonism. [22] VP patients usually present with lower-body parkinsonism, including impaired gait, instability, difficulty with maintaining gait, and frequent episodes of freezing. [4] Zijlmans et al. proposed a diagnostic criteria that includes the two components of VP: cerebrovascular disease and parkinsonism. The cerebrovascular disease presents as focal radiological signs that can be clinically correlated with the presentation of stroke.

Managing VP relies on controlling vascular risk factors. [4] However, Miguel-Puga et al. proposed a management strategy that includes the following four components: levodopa followed by transcranial magnetic stimulation, then vitamin D and LP. [23] The selection of these treatment components is based on prior clinical studies of parkinsonism patients. In all five patients in which transcranial magnetic stimulation was tested, motor function significantly improved. [24] Similarly, vitamin D has been shown to prevent falls. [25] LP, however, appears to be less effective with only five out of 40 patients receiving LP in a pilot study showing significant subjective improvement. [26] The case presented herein demonstrates the overlapping clinical presentation of iNPH and VP along with the response to lumber puncture.

\section{Case Report}

\subsection{Case Presentation}

A 71-year-old male was admitted electively through the clinic for possible VP. He had two prior lacunar strokes that were resolved without residual deficits. His additional history included having diabetes for 20 years, which was controlled through insulin and oral hypoglycemic drugs. He also had stage 3 chronic kidney disease (for which he was receiving treatment), hypertension, benign prostatic hyperplasia, and a history of right-eye cataract surgery. He had no history of fever, headache, neck pain, visual disturbance, speech abnormality, facial asymmetry, decreased hearing, tinnitus, weakness, numbness, altered sensorium, or depressed mood. Eight months before admission, the patient developed a progressively worsening magnetic gait and associated frequent falls. This led to dependence on a cane and limitations in his daily activities, such as requiring assistance using the restroom. Four months after the appearance of his gait disturbance he started to experience urinary urgency and increased frequency.

Upon examination, the patient was alert; properly oriented with respect to time, place, and person; and scored 20 out of 30 points on the Montreal Cognitive Assessment (MoCA). Cranial nerve examination was unremarkable apart from a diminished gag reflex and prior right-eye cataract surgery. Muscle strength was normal with a score of five out of five for both upper and lower limbs. He had normal tone with delayed ankle reflexes. Sensation was intact. Despite otherwise normal findings, he was unable to walk without a walking aid. Gait initiation was hesitant. Once moving, his gate was wide-based, magnetic, and with decreased arm swing. An objective assessment by a physiotherapist was performed.

\subsection{Investigation}

Magnetic resonance imaging (MRI) showed dilated ventricles (Figures 1 and 2), while the magnetic resonance angiography and magnetic resonance venography were unremarkable. Similarly, the nerve conduction study was normal. Urea $(11 \mathrm{mmol} \backslash \mathrm{L})$ and creatinine $(144$ umol $\backslash \mathrm{L})$ were elevated, but normalized after fluid infusion. Otherwise, laboratory findings were normal, including ceruloplasmin, parathyroid hormone, thyroid stimulating hormone, and vitamins D and B12. The patient underwent an objective gait assessment. Subsequently, an aseptic LP with an opening pressure of $12 \mathrm{~cm} \mathrm{H}_{2} \mathrm{O}$ drained $50 \mathrm{~mL}$ of CSF that was sent to the laboratory for haematology and biochemistry analysis, which were normal. 


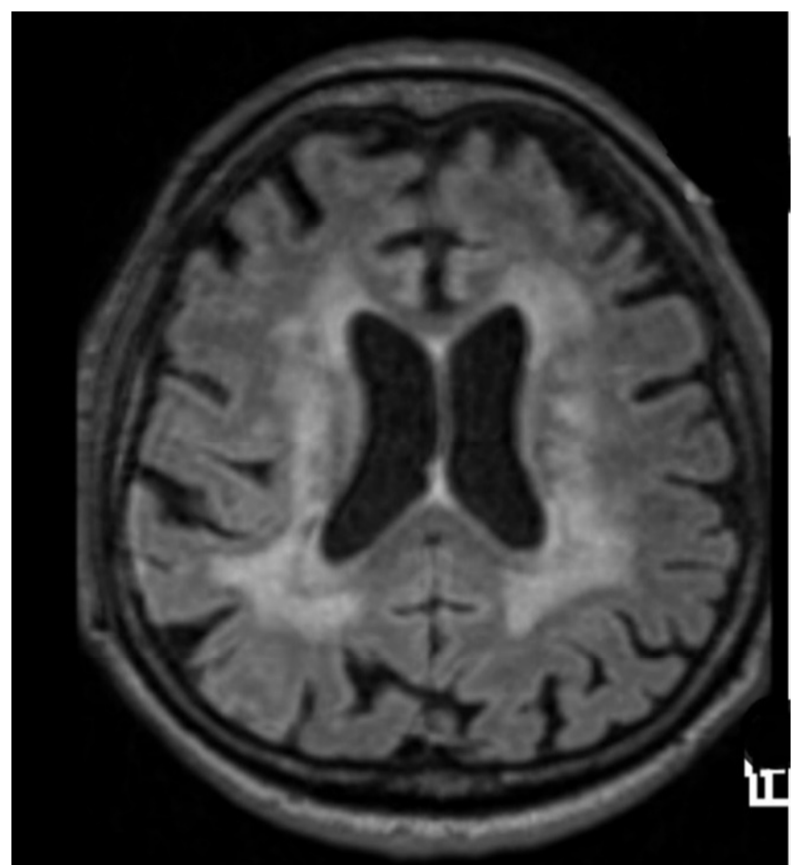

Figure 1. Axial FLAIR sequence shows symmetrically dilated ventricles with transependymal CSF flow $(90 \times 99 \mathrm{~mm}, 300 \times 300 \mathrm{DPI})$.

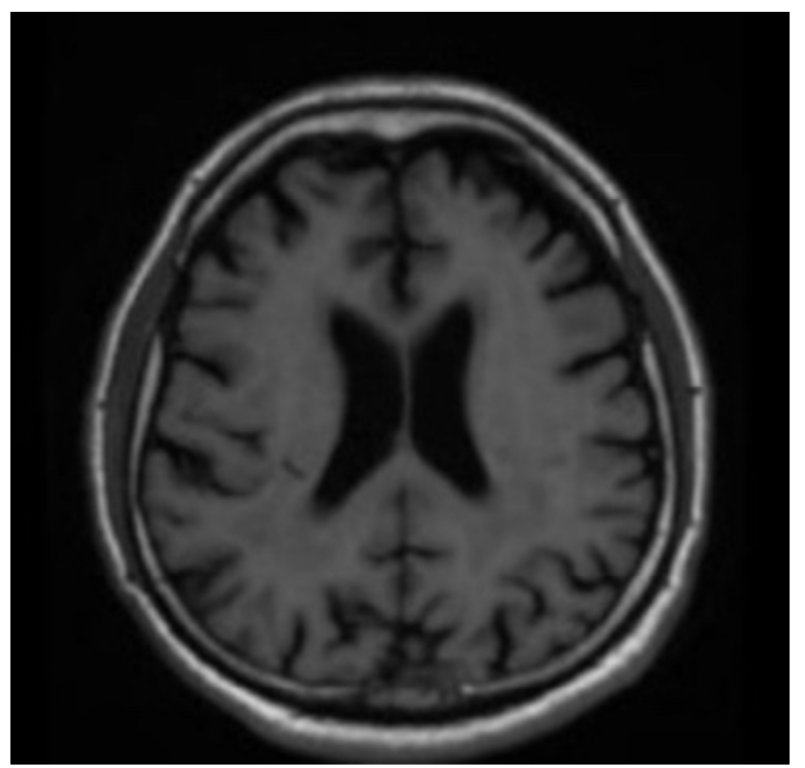

Figure 2. Axial T1 image shows symmetrically dilated ventricles with the associated Sylvain fissure and focally dilated sulci $(90 \times 61 \mathrm{~mm}, 300 \times 300$ DPI).

\subsection{Differential Diagnosis}

1. Idiopathic normal pressure hydrocephalus.

2. Vascular parkinsonism.

\subsection{Treatment}

Two months of treatment with levodopa $(110 \mathrm{mg}$, four times per day) did not improve symptoms. LP was performed to aid in diagnosis and as a therapeutic intervention, yielding improvement in the patient's symptoms and a minimal cognitive improvement (MoCA score increased to 24 out of 30).

\subsection{Outcome and Follow-up}

The patient's gait was assessed pre- and post-LP using objective and subjective measures, similar to a prior German study. [27] Objective measurements included four tasks, with each performed twice. The tasks included two speed tests (single-task preferred walking speed and maximal walking speed) and two cognitive dual-task tests (one using working memory to count backwards by serial 7 subtractions while walking and one task using semantic memory and verbal fluency to enumerate animal names while walking). A clinical hallmark of iNPH is a walking pace reduced to approximately 50\% that of healthy subjects. [27, 28] Patient performance after LP was compared to that before LP, and his speed showed slight improvement. Subjective assessments were conducted 30 minutes and 20 hours after LP by asking the patient to evaluate his gait with the question, "Do you think that your gait performance has improved after LP?" Qualitative and quantitative gait assessments can be very helpful to provide standardized and comparable data for clinicians to observe patient response to LP and should be routinely performed as an outcome measure following LP in patients with iNPH. [29]

\section{Discussion}

This is an interesting case of iNPH that was initially misdiagnosed as VP because of the patient's history of strokes and the similar presentation of these two conditions. Because the patient did not respond to levodopa $(110 \mathrm{mg}$, four times per day), idiopathic PD was excluded as a diagnosis and VP appeared unlikely. Furthermore, his symptoms improved after LP, which ruled out VP and led to the consideration of iNPH as an alternative diagnosis. This diagnosis was further supported by the patient having 2 out of the 3 components of the Hakim triad: gait disturbances and urinary symptoms. However, Alberto $\mathrm{J}$ et al. found that the Hakim triad was unable to differentiate between the two disorders, as urinary incontinence and cognitive impairment are nonspecific and can occur in the late stages of VP. An additional case of overlapping symptoms of iNPH and VP comes from a recent report by Cucca et al. in which a 79year-old female was thought to have PD according to the UKPDSBB criteria and started appropriate treatment. [30] Over the following months, the patient's mental status, gait, and balance deteriorated, with her MoCA decreasing to 22 out of 30. Increasing her dose of levodopa did not improve her symptoms. At this point, iNPH was considered as a possible diagnosis, and a repeat brain MRI showed increased ventricular size. After LP, her gait and neuropsychological assessments improved. Lee et al. reported a similar case of VP overlapping with iNPH, in which the patient experienced improvements in gait, cognition, and urinary symptoms over 7 days after LP. [31]

To maximize patient outcomes and optimize their care, distinct diagnostic criteria for both VP and iNPH need to be established, with the possibility of defining a new subtype 
that may have characteristics of both conditions. It is not uncommon for patients to be initially diagnosed as having $\mathrm{VP}$, and then for the diagnosis to be replaced by iNPH when they show no response to levodopa. The coexistence of iNPH with other pathologies (such as $\mathrm{VD}, \mathrm{AD}$, and others) makes diagnosis difficult. iNPH is a potentially treatable disease; however, the presentation is sometimes atypical and may overlap with other conditions. iNPH is still poorly understood, and the underlying aetiologies that may lead to both iNPH and VP need to be further elucidated. However, they may share the same underlying mechanism of altered transependymal CSF drainage. Gait disturbances resulting from VP can respond to CSF shunting, which is the dominant therapeutic approach for iNPH. [9] This indicates that VP and iNPH may not always represent two separate entities and that a novel disorder might exist in the overlap between these two conditions, which would require a novel approach in terms of diagnosis and management.

The utility of radiological findings in diagnosing iNPH is subject to debate, but some may be specific for iNPH. Dilated ventricles, Sylvian fissure, and basal cistern are present in most patients with iNPH. Dilatation of the aqueduct and fourth ventricle may be the most specific, as these are uncommon in patients with other relevant diseases. Sylvian fissure dilation may be somewhat specific because it is larger in patients with iNPH than with Alzheimer. Dilation of the basal cistern is not diagnostic, as it has been shown to occur across multiple disease states. Based on these findings, increased ventricular volume and decreased suprasylvian volumes could be a very specific diagnostic criterion for iNPH. In addition, enlarged basal cisterns and Sylvian fissures and focally dilated sulci further support the diagnosis of iNPH. [32]

MRI is the most precise test to detect the diagnostic features of iNPH. The International and the Japanese iNPH Guidelines proposed key imaging findings to diagnose iNPH and identify shunt-responsive patients. [33] Although some have questioned its utility, the Evans Ratio has been recently shown to be reliable for determining ventricular enlargement, without the need for high cost, lengthy, and technically difficult computer programs that may not be available in all healthcare settings. [33] Enlarged ventricles associated with large basal cisterns and Sylvian fissures and focally dilated sulci supports a diagnosis of shunt-responsive iNPH and should not be disregarded as a minor sign of cerebral atrophy. In normal aging and degenerative diseases, sulci dilation is more generalized and occurs to a comparable degree as that of other brain regions. [32, 33] Other MRI findings in typical iNPH include a callosal angle (CA) between $40^{\circ}$ and $90^{\circ}$; wide temporal horns; and callosal upward elevation, stretching, and thinning, with the latter two predicting positive shunt response. As tools to compliment MRI, single photon emission computed tomography and positron emission tomography may reflect reduced cerebral blood flow and metabolism that can occur in iNPH. [33] These imaging tools may also reveal improved regional cerebral metabolic rates in shunt-responsive patients. [33] Based on findings from previous radiological studies, we conclude that imaging plays a significant role in diagnosing iNPH while excluding other possible differential diagnoses. In addition, imaging may also predict shunt outcomes and be useful for outcome monitoring.

\section{Conclusion}

Idiopathic normal pressure hydrocephalus (iNPH) is the most common form of hydrocephalus in adults. It is characterized by dilated ventricles and the Hakim triad, which consists of gait disturbance, cognitive impairment, and urinary incontinence. Furthermore, iNPH is frequently mistaken or overlap with some conditions such as vascular parkinsonism, such overlap requires further elucidation. iNPH and VP are two treatable conditions in which the differential diagnosis between the two entities is challenging. We recommend further studies to provide diagnostic clinical criteria, neuroradiological and neurophysiological biomarkers to enhance diagnostic accuracy between these two conditions.

\section{References}

[1] Williams MA, Malm J. Diagnosis and Treatment of Idiopathic Normal Pressure Hydrocephalus. Continuum (Minneap Minn) 2016; 22: 579-99.

[2] Virhammar J, Laurell K, Cesarini KG, et al. The callosal angle measured on MRI as a predictor of outcomes in idiopathic normal-pressure hydrocephalus. J Neurosurg 2014; 120: 178184.

[3] Korczyn AD. Vascular parkinsonism - characteristics, pathogenesis and treatment. Nat Rev Neurol 2015; 11: 319-26.

[4] Bovonsunthonchai S, Witthiwej T, Ngamsombat C, et al. Effect of spinal tap test on the performance of sit-to-stand, walking, and turning in patients with idiopathic normal pressure hydrocephalus. Nagoya J Med Sci 2018; 80: 53-60.

[5] Chen Y-C, Chiang S-W, Chi C-H, et al. Early Idiopathic Normal Pressure Hydrocephalus Patients with Neuropsychological Impairment Are Associated With Increased Fractional Anisotropy in the Anterior Thalamic Nucleus. Medicine 2016; 95: e3636.

[6] Ghosh S, Lippa C. Diagnosis and Prognosis in Idiopathic Normal Pressure Hydrocephalus. Am J Alzheimers Dis Other Demen 2014; 29: 583-9.

[7] Blomsterwall E, Svantesson U, Carlsson U, et al. Postural Disturbance in Patients with Normal Pressure Hydrocephalus. Acta Neurol Scand 2000; 102: 284-291.

[8] Maalouf M, Ringman J, Shi J. An Update on the Diagnosis and Management of Dementing Conditions. Rev Neurol Dis 2011; 8: e68-e87.

[9] Shprecher D, Schwalb J, Kurlan R. Normal Pressure Hydrocephalus: Diagnosis and Treatment. Curr Neurol Neurosci Rep 2008; 8: 371-376. 
[10] Abdool K, Seegobin K, Ramcharan K, et al. Neurosyphilis With Normal Pressure Hydrocephalus and Dementia Paralytica: Serial Clinical, Laboratory and Radiological Correlations in the 21st Century. Neurol Int 2016; 8: 6812.

[11] Halperin JJ, Kurlan R, Schwalb JM, Cusimano MD, Gronseth G, Gloss D. Practice guideline: Idiopathic normal pressure hydrocephalus: Response to shunting and predictors of response: Report of the Guideline Development, Dissemination, and Implementation Subcommittee of the American Academy of Neurology. Neurology 2016; 86 (23): 793.

[12] Hailong F, Guangfu H, Haibin T, et al. Endoscopic third ventriculostomy in the management of communicating hydrocephalus: a preliminary study. $J$ Neurosurg 2008; 109: 923-30.

[13] Halperin JJ, Kurlan R, Schwalb JM, et al. Practice guideline: Idiopathic normal pressure hydrocephalus: Response to shunting and predictors of response: Report of the Guideline Development, Dissemination, and Implementation Subcommittee of the American Academy of Neurology. Neurology 2015; 85: 2063-2071.

[14] Ishikawa M, Hashimoto M, Mori E, Kuwana N, Kazui H. The Value of the Cerebrospinal Fluid Tap Test for Predicting Shunt Effectiveness in Idiopathic Normal Pressure Hydrocephalus. Fluids Barriers CNS 2012; 9 (1): 1.

[15] Lim TS, Yong SW, Moon SY. Repetitive Lumbar Punctures as Treatment for Normal Pressure Hydrocephalus. Eur Neurol 2009; 62: 293-297.

[16] Critchley M. Arteriosclerotic parkinsonism. Brain 1929; 52: 23-83.

[17] Eadie MJ, Sutherland JM. Arteriosclerosis in Parkinsonism. $J$ Neurol Neurosurg Psychiatry 1964; 27: 237-240.

[18] Parkes JD, Marsden CD, Rees JE, et al. Parkinson's disease, Cerebral Arteriosclerosis, and Senile Dementia. Clinical Features and Response to Levodopa. Q J Med 1974; 43: 4961.

[19] Zijlmans JC, Daniel SE, Hughes AJ, et al. Clinicopathological Investigation of Vascular Parkinsonism, Including Clinical Criteria for Diagnosis. Mov Disord 2004; 19: 630-40.

[20] Benamer HT, Grosset DG. Vascular Parkinsonism: A Clinical Review. Eur Neurol 2009; 61: 11-5.

[21] Thanvi B, Lo N, Robinson T. Vascular Parkinsonism - an Important Cause of Parkinsonism in Older People. Age Ageing 2005; 34: 114-119.
[22] van Swieten JC, van den Hout JH, van Ketel BA, et al. Periventricular Lesions in the White Matter on Magnetic Resonance Imaging in the Elderly. A Morphometric Correlation with Arteriolosclerosis and Dilated Perivascular Spaces. Brain 1991; 114: 761-774.

[23] Miguel-Puga A, Villafuerte G, Salas-Pacheco J, et al. Therapeutic Interventions for Vascular Parkinsonism: A Systematic Review and Meta-analysis. Front Neurol 2017; 8: 481.

[24] Yip CW, Cheong PW, Green A, et al. A Prospective Pilot Study of Repetitive Transcranial Magnetic Stimulation for Gait Dysfunction in Vascular Parkinsonism. Clin Neurol Neurosurg 2013; 115: 887-91.

[25] Sato Y, Iwamoto J, Honda Y, et al. Vitamin D Reduces Falls and Hip Fractures in Vascular Parkinsonism but not in Parkinson's Disease. Ther Clin Risk Manag 2013; 9: 171-176.

[26] Ondo WG, Chan LL, Levy JK. Vascular Parkinsonism: Clinical Correlates Predicting Motor Improvement After Lumbar Puncture. Mov Disord 2001; 17: 91-7.

[27] Schniepp R, Trabold R, Romagna A, et al. Walking Assessment after Lumbar Puncture in Normal-Pressure Hydrocephalus: A Delayed Improvement over 3 Days. $J$ Neurosurg 2017; 126: 148-157.

[28] Wikkelsø C, Andersson H, Blomstrand C, et al. The Clinical Effect of Lumbar Puncture in Normal Pressure Hydrocephalus. J Neurol Neurosurg Psychiatry 1982; 45: 6469.

[29] Stolze H, Kuhtz-Buschbeck JP, Drücke H, et al. Gait Analysis in Idiopathic Normal Pressure Hydrocephalus - Which Parameters Respond to the CSF Tap Test? Clin Neurophysiol 2000; 111: 1678-1686.

[30] Cucca A, Biagioni MC, Sharma K, et al. Comorbid Normal Pressure Hydrocephalus with Parkinsonism: A Clinical Challenge and Call for Awareness. Case Rep Neurol Med 2018; 2018: 1-8.

[31] Lee H-W, Kang K, Choi D. Unusual Idiopathic Normal Pressure Hydrocephalus Patient with Marked Asymmetric Upper Body Parkinsonism. Ann Indian Acad Neurol 2016; 19: 245.

[32] Kitagaki H, Mori E, Ishii K, et al. CSF Spaces in Idiopathic Normal Pressure Hydrocephalus: Morphology and Volumetry. AJNR Am J Neuroradiol 1998; 19: 1277-84.

[33] Damasceno BP. Neuroimaging in Normal Pressure Hydrocephalus. Dement Neuropsychol 2015; 9: 350-355. 